\title{
Should Natural Gas Be Shipped or Stored to Supply Power Plants?
}

\author{
Seth Blumsack \\ Penn State University and Santa Fe Institute \\ sab51@psu.edu
}

\author{
Yucheng $\mathrm{Wu}$ \\ Penn State University \\ yzw124@psu.edu
}

\begin{abstract}
Following a series of winters featuring extreme cold episodes in the Northeastern U.S., power grid operators have engaged in exercises focused on assessing fuel deliverability to power plants, particularly natural gas. These studies have raised important issues and identified possible scenarios that could contribute to reliability problems during winter peaks, but have not evaluated the economics of specific solutions to winter-time fuel deliverability. This paper describes an expansion to a new modeling framework for gas and electric power transmission planning problems (the Combined Electricity and Gas Expansion, or CEGE model) that allows centralized or distributed natural gas storage to be evaluated alongside traditional planning alternatives such as transmission network expansion. Using a test system based on the gas and electric transmission topology in New England, we develop a a simple two-period gas storage model and use this model to evaluate economically valuable locations for distributed natural gas storage and compare the economic merits of increasing storage within New England versus expanding pipeline infrastructure to increase fuel deliverability to New England power plants within our test system. Initial simulations using this storage model suggest that the optimal placement for gas storage may be co-located with power plants to relieve binding pressure constraints in areas of the gas network close to gas-fired generation. Moreover, the economic consequences of extreme winter peak scenarios may be ameliorated at a lower cost with a mix of gas storage and pipeline expansions rather than via pipeline expansion alone.
\end{abstract}

\section{Introduction}

The emergence of low-cost natural gas has been responsible for a major shift in fuels utilization in many portions of the North American power grid. Natural gas is quickly taking a larer share of power generation, displacing coal and economically threatening some nuclear power facilities. Analyses of how this shift will impact the economics of power grid planning and operations, as well as reliability, have been mixed [1, 2, 3, 4, 5, 6]), with several studies suggesting that impacts on reliability and system operations should be minimal and others (particularly in light of extreme cold conditions during the winters of 2013/14 and 2016/17 in the U.S. Northeast) raising continued concerns about the dependence of the power grid on deliveries from the natural gas transmission infrastructure. Most notably, at least two Regional Transmission Organizations in the Northeastern U.S. (PJM and ISO-New England) have launched initiatives to assess "fuel security" - the impacts that gas delivery interruptions may have on power system operations [5,7]. Specific gas transmission upgrades to support the electric power grid have been proposed for portions of New England [8], and the fuel security analysis published by ISO New England considers scenarios with expanded natural gas storage and other technology solutions [7]. How different technological options for managing coincident winter peaks compare on economic grounds is, however, missing from the literature at present.

In this paper, we describe and implement a modification to an existing analysis tool for gas-grid problems, the Combined Electric and Gas Expansion (CEGE) planning model $[9,10,11,12]$. The CEGE model is an open-source optimization-based modeling framework for joint planning and operations problems involving interdependent natural gas and electric power transmission systems. We modify the CEGE framework to include a simple two-stage gas storage model in order to understand the relative economics of using gas storage (centralized or distributed) versus additional pipeline infrastructure to ensure gas deliverability to power plants specifically. Our storage model allows the optimization engine to choose the most economically beneficial locations for gas storage to supply power-plant needs, although the dispatch of storage is more stylized, with a representative off-peak period in which gas is withdrawn from the transmission network to fill storage; and a representative on-peak period in which gas is injected into the transmission network or used on-site to meet local demands. In this sense we do not claim that the gas dispatch element of the model is optimal (as in [13, 14, 15], 
but this modeling feature is currently in development as an ongoing area of research.

The modeling experiments are implemented on the gas-grid test system described in $[10,12]$, which features a realistic network topology for the gas and electric power systems in the Northeastern U.S., particularly New York and New England. The value of the test system is not to reproduce historical market outcomes with precision, but to provide a realistic topology and gas transport situation for a test system that depends on natural gas for heating and power generation during the winter season but have little or no indigenous natural gas resources. The test system itself features a large consuming area whose natural gas transmisson topology is similar to that of the gas transmission system covering New York and New England, and a gas production area in reasonable proximity, similar to how the Marcellus Shale producing area could transport large production volumes to New York and New England. In this sense, the contribution of the present paper is to demonstrate an analytical framework that is able to evaluate tradeoffs in the mode of transportation and storage for energy commodities while considering some of the realistic and computationally difficult constraints (such as pressures on the natural gas system) present in large-scale energy infrastructures. Previous work on similar decision problems has concluded that the movement of fuels rather than electricity tends to be more efficient over long distances [16] and analysis of technology tradeoffs in the design of energy and water systems $[17,18,19]$.

To make comparisons between the alternatives for supplying fuel to gas-fired power plants during winter peak demand scenarios, we run several different simulations. We first simulate a scenario where the gas transmission network is hardened to an extent that would prevent large price spikes during extreme demand events while meeting all demand. We find that the network investments required to avoid these price spikes would be substantial, around 40 percent higher than the cost of the investment plan that balances capital costs and network operational costs [12]. This first simulation assumes no additional storage investment. We then simulate a scenario where a system planner could choose between investments in additional gas transmission links or peakshaving gas storage facilities $[20,21]$ at specific locations. Two findings of particular interest emerge from these simulations. The first is that for the specific purposes of ensuring fuel supply at gas-fired power plants during winter peak demand periods without substantial price spikes, the optimal placement for distributed peak-shaving gas storage appears to be co-location with natural gas power plants in ways that relieve localized pressure constraints. We do acknowledge that such co- location may be cost-minimizing but can face real siting acceptance challenges. The second is that a portfolio of distributed peak-shaving gas storage and some pipeline expansion can avoid price spikes at a lower cost than with pipeline expansion alone.

In Section 2 we provide an overview of the CEGE model and describe the gas storage model that we develop. The results of our computational experiments are described in Section 3. We conclude in Section 4 with some thoughts on future directions for research on the tradeoffs between technology choices in interdependent gas-electric systems.

\section{The CEGE Model Structure}

CEGE is a large-scale multi-level optimization problem including constraints and variables associated with modeling the non-convex physics of electric power and natural gas systems, modeling expansion options and costs, modeling heat-rate curves, and incorporating power generation costs. This section provides a brief description of the model's structure; a more complete mathematical description of the model formulation can be found in [12].

The overall structure of the model is shown in Figure 1. The highest level of the model is an optimizationbased least-cost planning objective, which assumes a single decision-maker for electric power transmission expansion; natural gas transmission expansion; or both. This decision-maker is assumed to choose a set of network expansions including new gas or electric transmission lines and new power plants, in order to minimize the total cost of network expansion and network operations. This paper will provide an example of how new gas storage may be modeled within the CEGE framework, but the model as described here does not itself optimize natural gas storage (this modeling enhancement is currently in progress). New gas wells are also not included as explicit decision variables in the modeling framework.

The lower levels of the CEGE model consist of two modules that capture the operation of gas and power transmission systems. Each of the two transmission systems is assumed to be controlled by a single centralized operator. The power grid module consists of an AC Optimal Power Flow (OPF) in which the grid operator chooses dispatch levels for power plants in order to minimize total operational costs, subject to various network constraints. The natural gas transmission module involves choosing compression settings and flow directions to ensure desired gas deliveries. Gas demands for uses other than power generation are assumed to be fixed and price-inelastic, while gas demands for power generation are price-elastic and are driven by demands from 


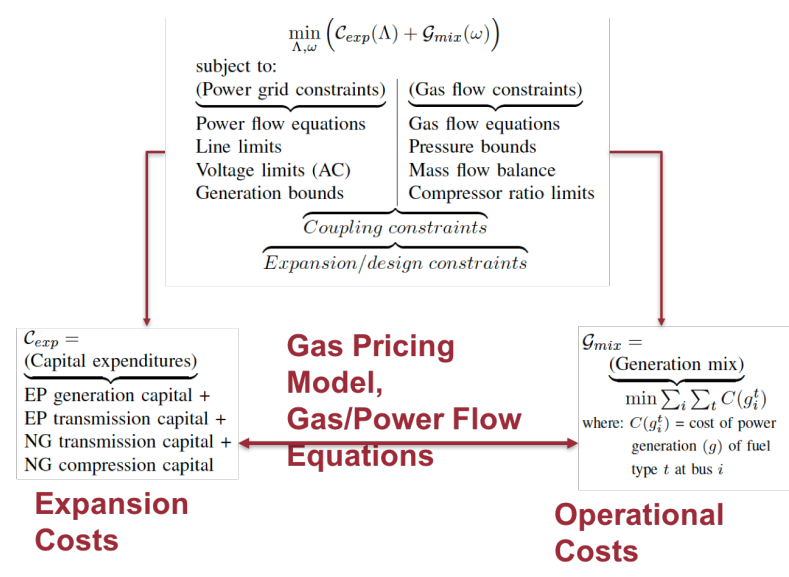

Figure 1: The Structure of the CEGE Model

the power grid module.

\subsection{A Simple Two-Period Gas Storage Model}

An innovation to the CEGE framework developed in this paper is the inclusion of a simple two-period gas storage model. Adapting the CEGE model to incorporate injections and withdrawals from gas storage involves two steps. First, the operational modules of the CEGE model (the lower levels of the model in Fig. 1) are expanded to contain two types of representative periods for both the electricity and natural gas systems. We refer to the first type of representative period as the "off-peak" period, and we assume that gas is injected into peak-shaving storage units in all off-peak periods. The demand profile for electricity and natural gas during the off-peak period is the base-case demand profile from the Northeastern U.S. test system described in $[11,12]$. Gas storage locations are modeled as price-inelastic consumers of natural gas during the off-peak period.

We refer to the second type of representative period as the "peak" period, and we assume that gas is withdrawn from storage during all peak periods. The demand profile for electricity and natural gas during the peak period inflates electricity demand by 35 percent at each electric load in the network, and inflates natural gas demand by 400 percent at each gas consumption location in the network. (This corresponds to the 1.35 power stress case and 4.0 gas stress case in [12].) Peak-shaving gas storage locations are modeled as suppliers of natural gas during the off-peak period, and withdrawals from gas storage during the peak period are constrained to be no greater than the injections during the off-peak period. In other words, our simple gas storage model assumes that all storage units are empty during the off-peak period, but the storage facilities do not need to be com- pletely emptied during the peak period. The amount of gas withdrawn from storage is a parameter of interest on which we perform a sensitivity analysis.

The objective function of the CEGE framework is modified to add gas storage locations and costs to the expansion portion of the objective function, and to add operational cost terms for the off-peak and peak periods, as follows:

$$
\begin{gathered}
\min \quad \sum_{a \in A^{e}} \kappa_{a}^{e} z_{a}^{e}+\sum_{a \in A_{p}^{g}} \kappa_{a}^{g} z_{a}^{g}+\sum_{n \in N^{g}} \kappa_{n}^{s} S_{n}+ \\
\sum_{\zeta \in Z} Z(\zeta)+ \\
{\left[\sum_{i \in \Gamma} \mu_{1}^{i}+\mu_{2}^{i} p_{i}^{g, o p k}+\mu_{3}^{i}\left(p_{i}^{g, o p k}\right)^{2}\right]+} \\
{\left[\sum_{i \in \Gamma} \mu_{1}^{i}+\mu_{2}^{i} p_{i}^{g, p k}+\mu_{3}^{i}\left(p_{i}^{g, p k}\right)^{2}\right]}
\end{gathered}
$$

Equation (1) follows the notation in [12]. The expansion terms of the CEGE objective function are in the first line of (1). $A^{e}$ and $A_{p}^{g}$ are the sets of edges in the electric and gas transmission system, $N^{g}$ is the set of junctions (nodes) in the gas transmission system, $\kappa_{a}^{e}$ and $\kappa_{a}^{g}$ represent the the capital costs for electric and gas transmission expansions along edges $e$ in the electric power system and $g$ in the gas transmission system (which includes new edges as well as expansions of existing edges in both the electricity and gas systems), $\kappa_{s}^{n}$ is the expansion cost for gas storage located at gas junction $n, S_{n}$ is the quantity of gas storage built at gas junction $n$, and $z_{a}^{e}$ and $z_{a}^{g}$ are binary expansion variables along edges $e$ and g.

The second line in (1) is the gas cost equation. $Z$ is the set of pricing zones in the gas network, and $Z(\zeta)$ is the pricing function for natural gas as described in [12].

The third and fourth lines in (1) are the power generation cost equations for the off-peak period (indexed by $o p k$ ) and the peak period (indexed by $p k$ ). $\Gamma$ is the set of power generation nodes in the electrical network, the $\mu^{i}$ are cost coefficients, and the $p_{i}^{g}$ terms represent the level of power output at the $i$ th generator.

The only other changes needed to the CEGE framework to accommodate this simple storage model involve some additional constraints in the natural gas module. The capacity of the storage unit is modeled by a set of constraints limiting injections (consumption during the off-peak period) and withdrawals (supply to the gas transmission system during the peak period). These values are taken from [21], although we do not impose the pressure constraints from that analysis. Additionally, the storage modification to the CEGE model does not really optimize the operation of storage once the model chooses to construct storage in a specific location. An injection scenario is defined that sets the amount of gas injected at the storage location during the off-peak period. Injection into gas storage is thus modeled in an 
equivalent way as other price-inelastic natural gas demand in the CEGE framework. Withdrawal scenarios are defined by constraints limiting the amount of gas supplied from storage.

We emphasize that this storage model is a starting point that is fairly stylized, particularly when applied to a peak-shaving scenario that may involve drawdowns from storage that vary over a several-hour time horizon. The modified CEGE objective function in (1) does choose the optimal storage locations from a candidate set of potential storage locations, but the operation of gas storage is not determined through any optimization or equilibrium framework. Additionally, the two periods in our model are linked through the storage constraints, but are not intended to represent the faster dynamics of gas flow as described in [22]. Improving the storage model is an active area of research, but even with the stylized storage model in the present paper we are able to describe some interesting results related to the economics of gas transmission investments driven by peak time demand considerations.

\section{Numerical Results}

In this section, we describe the numerical results of applying the CEGE to the gas-grid test system described in $[11,12]$. We use the CEGE model (with joint expansion planning for electricity and gas) to compare two strategies for mitigating the market price impacts of extreme coincident peak demand periods for electricity and natural gas (a Polar Vortex type case). Relative to the demand profile described in [12], which we use as our base-case or off-peak demand profile, our Polar Vortex type case is a coincident winter peak where electricity demand is 35 percent higher than in the base case and natural gas demand is four hundred percent higher than in the base case. We assume a levelized cost for distributed natural gas storage of $\$ 4 / \mathrm{mmBTU}$, which is consistent with the cost of LNG for peak-shaving purposes if that peak-shaving cost were levelized over all gas demand in the network [20,21].

The first strategy permits network expansion through increased pipeline capacity only. The second strategy uses the storage model from Sec. 2 in which distributed gas storage units are filled during an off-peak demand period for both gas and electricity and this storage can thus be injected into the gas transmission network during the peak demand period (again, the peak demand period in the storage model represents a coincident peak for both electricity and natural gas). We impose an additional constraint on this analysis that gas prices rise no more than $10 \%$ relative to base case levels. The idea here is to simulate the kinds of investments that would be necessary to avoid the most extreme economic consequences of coincident peak demands for electricity and natural gas while still maintaining a feasible solution that meets demands.

In our simulations, we allow any existing gas or electric transmission corridor to expand its capacity and allow some new gas pipeline corridors to be added. The options for new candidate corridors are defined based on proposed gas pipeline expansions in the northeastern U.S. These expansions would all facilitate additional gas shipments from the Marcellus Shale producing area in Pennsylvania to demand centers in the Mid-Atlantic, New York, and New England. The specific expansions are listed below, with their geographic locations and names of the proposed pipeline expansions on which our modeled expansions are based.

1. NG1: A new east-west link across PA (expansion of the Texas East line)

2. NG2: A new link increasing delivery into eastern PA and NJ (the Penn East line)

3. NG3: New links from north-central PA to the south and northeast (the Transco expansions)

4. NG4: A new link from north-central PA going north towards NY (the Marc II line)

5. NG5: A new link from PA to NY (the Constitution line)

6. NG6: A new link increasing delivery from northcentral PA to southeastern PA (the Sunbury line)

In our simulations, some new pipeline corridor expansions were chosen as lower-cost alternatives to expanding existing corridors to meet higher gas demand and to ameliorate gas price spikes. This is driven by our peak gas case assumption; at lower levels of natural gas demand no new pipeline links were created (although as described in [12], some existing pipeline corridors were expanded at lower levels of gas demand). The new pipeline corridors that were chosen by the model did vary by the power system stress case, as shown in Table 1. At lower levels of power system stress, the model adds combinations of new gas corridors NG3 and NG6, which would expand gas deliveries from the Marcellus producing areas to areas of higher gas prices in New York and the Mid-Atlantic coast. At higher levels of power system stress, the model adds NG2 and NG6, which expand gas delivery capability into the MidAtlantic coastal areas rather than expanding capacity into New York.

\subsection{Avoiding Extreme Price Spikes}

Our first set of simulations seeks to avoid extreme price spikes using only pipeline capacity expansions. The results are described in Table 2, which presents the 
Table 1: New gas transmission corridors built in high gas stress cases.

\begin{tabular}{|l|l|}
\hline Power System Stress Case & New Gas Transmission \\
\hline 1.0 PS & NG3 \\
1.1 PS & NG3 and NG6 \\
1.25 PS & NG2 and NG6 \\
1.3 PS & NG2 and NG6 \\
\hline 1.35 PS & NG2 and NG6 \\
\hline & \\
\hline & \\
\hline & \\
\hline
\end{tabular}

Figure 2: Gas and electric expansion plan to avoid gas price spikes larger than $10 \%$ during periods of extreme gas and electric demands.

network expansion results when the limit is set to $100 \%$, $10 \%, 5 \%, 1 \%$, and $0 \%$ of the penalty for the $4.0 \mathrm{GS}$ and 1.35 PS problem. A visual set of results showing the locations of gas and electric network expansions in the case where gas price spikes are limited to $10 \%$ is shown in Figure 2. The variables $z^{e}$ and $z^{g}$ represent the number of electric and gas transmission expansions. Interestingly, the gas network must be expanded by another $40 \%$ compared to the results described in [12] to avoid the types of price spikes that were observed during the polar vortex, as soon as the penalty is limited to $10 \%$ or less. The electricity network is not affected in this case, consistent with the observation that gas transmission was scarce during the polar vortex, not gas supply per se. Our results do suggest that a policy of infrastructure hardening to avoid the economic consequences of weather-driven demand spikes in the electricity and natural gas systems would be costly, relative to the benefits of spot gas price reduction, and would need to be concentrated in enhancing fuel delivery rather than electric power delivery.

Figure 3 shows the locations of distributed gas storage units that could be deployed in order to reduce the

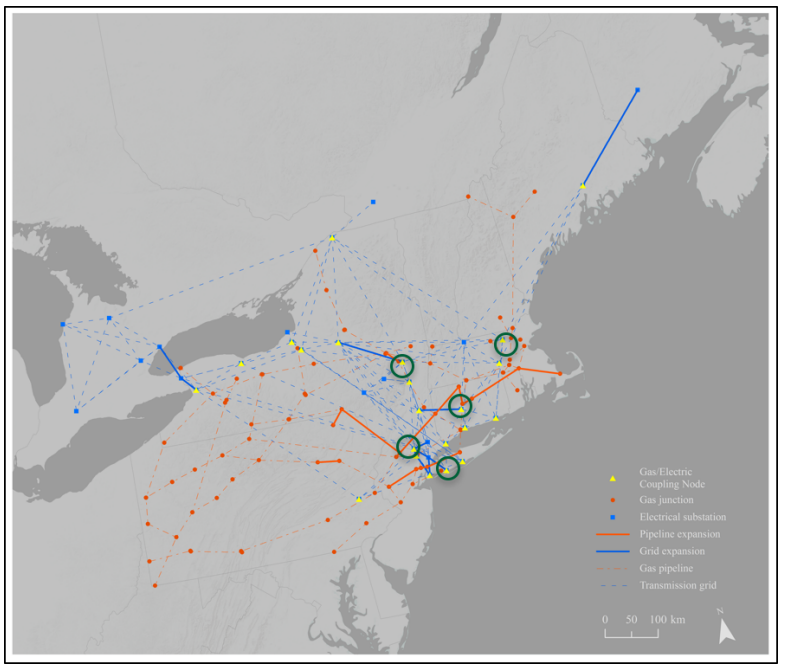

Figure 3: Distributed gas storage at interconnection locations with gas-fired power plants to avoid gas price spikes larger than $10 \%$ during periods of extreme gas and electric demands.

network expansions needed to limit gas price spikes to no more than $10 \%$ of the base case gas price level during the gas-electric coincident peak demand period. These locations were determined based on identification of the gas network nodes with the highest value of pressureconstraint relief in a simulation with increased demand for electricity and gas but no additional gas or electric transmission investment. The value of the pressure penalty cost (our measure of gas system congestion as outlined in [12]) was the highest in these particular locations. Interestingly, the locations with the highest pressure penalty costs were those where one or more gasfired power plants interconnected with the gas transmission system in our test network. Even at a levelized cost of $\$ 4$ per mmBTU (which translates into $\$ 96,000$ per mmscfd) the use of distributed gas storage at major gasfired generation locations in the eastern portion of our test system (which would correspond geographically to New York and New England - the downstream ends of major gas transmission paths) halves the additional gas transmission investment needed to avoid large gas price spikes during extreme demand events.

CEGE simulations run using the storage model suggest that gas storage and gas transmission may be substitutable to some extent [20,7]. Adding the option for distributed gas storage still requires more pipeline investment to avoid large gas price spikes (relative to a scenario where demand must be served but price spikes are tolerated), but fewer pipeline investments than in a planning scenario where pipeline expansions are the only option. To gain additional insight on the potential 
Table 2: Avoiding Extreme Price Spikes via Pipeline Expansion.

\begin{tabular}{|l|cc|cc|cc|cc|cc|}
\hline & \multicolumn{2}{|c|}{1.0 PS } & \multicolumn{2}{|c|}{1.1 PS } & \multicolumn{2}{|c|}{1.25 PS } & \multicolumn{2}{|c|}{1.3 PS } & \multicolumn{2}{|c|}{1.35 PS } \\
& $z_{e}$ & $z_{p}$ & $z_{e}$ & $z_{p}$ & $z_{e}$ & $z_{p}$ & $z_{e}$ & $z_{p}$ & $z_{e}$ & $z_{p}$ \\
\hline $100 \%$ & 8 & 14 & 9 & 13 & 11 & 14 & 13 & 13 & 16 & 12 \\
$10 \%$ & 7 & 17 & 10 & 15 & 10 & 17 & 11 & 17 & 14 & 17 \\
$5 \%$ & 8 & 15 & 10 & 14 & 10 & 17 & 10 & 19 & 16 & 17 \\
$1 \%$ & 9 & 17 & 9 & 19 & 10 & 19 & 10 & 19 & 16 & 17 \\
$0 \%$ & 8 & 16 & 10 & 17 & 11 & 18 & 11 & 20 & 16 & 19 \\
\hline
\end{tabular}

for distributed gas storage to substitute for gas transmission specifically for the purpose of supporting gas delivery also conduct a sensitivity analysis on the amount of natural gas stored at the identified optimal storage locations (recall that the CEGE model described in the present paper chooses the best locations for gas storage, but the amount of gas stored and withdrawn is an adjustable parameter) to the amount of new natural gas pipeline infrastructure.

The sensitivity analysis is conducted by varying two parameters. First the utilization of gas storage investments is adjusted in the range of zero (no utilization even though the storage facilities exist) to full utilization based on the capacity of the distributed storage units. This analysis assumes that all storage facilities are utilized with the same level of intensity. Second, we vary the utilization of gas pipeline expansion options by derating the capacity of some expansions chosen by the CEGE model. For consistency with how we model the utilization of gas storage, we also describe the utilization of potential gas pipeline expansions in proportional terms, between zero (meaning that all pipeline investments are turned off) to full utilization. A pipeline utilization of 50 percent, for example, would mean utilizing half of the optimal chosen pipeline capacity.

The results of the sensitivity analysis are shown in Fig. ??. Panel (a) in Fig. ?? shows the relative change in total costs in the low gas price zone (the western portion of our Northeastern test system) while panel (b) shows the relative change in total costs in the high gas price zone (the eastern portion of the test system). Total cost here for a specific zone refers to the sum of investment costs for expansions in that zone, plus operating costs for gas and electric infrastructure in that zone. (Note that our cost measure here is potentially imperfect in that the costs of producing power or gas in one zone and moving it to the other zone are accrued in the producing zone rather than the consumption zone.) The contour lines indicate combinations of storage and pipeline utilization that yield identical changes in total zonal costs, relative to the portfolio of storage and pipeline utilization shown in Fig. 3.

Fig. ?? suggests that for the specific purpose of meeting power-plant natural gas demands during coincident winter gas and electric demand peaks, distributed gas storage may be a lower-cost option. Relative total costs tend to decline as additional storage is used relative to pipeline expansion. The primary reason for this finding may be that the sensitivity analysis is comparing gas storage specifically for power-plant fuel support versus large-scale pipeline infrastructure which may serve multiple demands. We believe, however, that the simulations are informative in light of concerns raised by some grid operators regarding the security of power-plant fuel delivery. The simulations here suggest that a policy of supporting fuel deliverability to power plants via largescale pipeline expansion may not be the lowest-cost solution.

\section{Conclusion}

This paper describes and demonstrates an extension of an existing framework for modeling expansion planning decisions in natural gas and electric power transmission systems (Combined Gas-Electric Expansion, or CEGE) to accommodate the option of gas storage for peak-shaving purposes as an alternative to additional gas transmission expansion. The model expansion described here can directly address current policy issues surrounding fuel security and planning for greater dependence of power grids on natural gas as a generation fuel. Incorporating distributed gas storage as an investment option has the potential to lower the costs of ensuring fuel security at gas-fired generation stations, relative to relying solely on additional gas transmission investment. Both pipelines and storage units, however, suffer similar economic problems of being useful only during highly specific and extreme coincident gas-electric demand peaks. Whether these extreme events should be handled entirely on the fuel supply side versus demand-side or energy efficiency solutions, depends on the frequency and severity of future extreme events. This is a potentially active area for research, just as demand response in electricity has become an active area of research and market expansion over the past several years. 

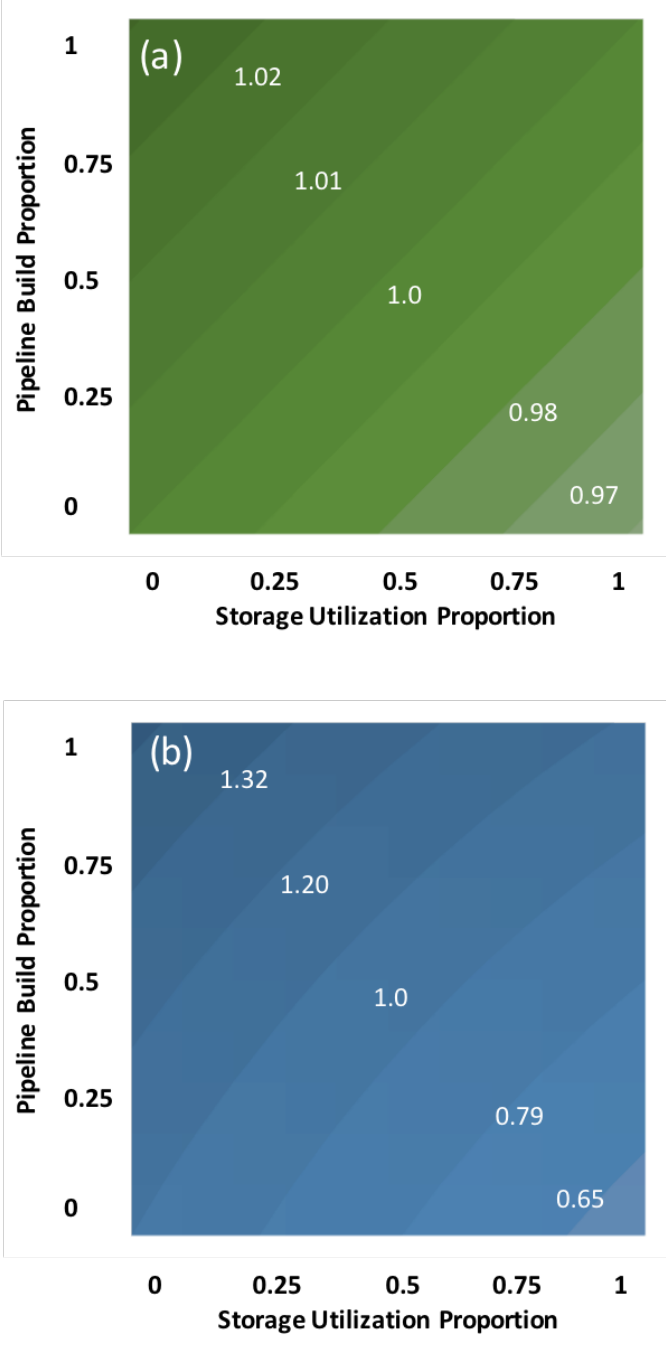

Figure 4: Change in the objective of the CEGE model, relative to the case shown in Fig. 3. The horizontal axis shows the proportion of storage used to supply gas to power plants during peak demand times. The vertial axis shows the proportions of additional gas delivery infrastructure utilized.

Our simulations suggest that for the specific purpose of meeting natural gas demands at power plants during coincident peak demand periods in both electricity and natural gas, peak-shaving gas storage co-located with natural gas power plants may be a cheaper alternative than additional long-distance gas transmission infrastructure specifically designed to meet gas-fired generation demands. Moreover, our system model suggests that co-location with power plants specifically offers an additional mechanism to reduce costs in the gas transmission network by addressing the source of pressure constraint violations during periods of high gas and electric demands. We should note that this result is driven entirely by the economic focus of the model, and does not speak to the siting difficulties that may be faced in expanding gas storage in some specific locations.

While the primary contribution of the present paper is to illustrate how the CEGE modeling framework may be adapted to consider intertemporal storage constraints in a computationally tractable way, the model formulation presented here is a work in progress and does have some limitations that we recognize. First, the gas storage model used here has the twin limitations of assuming a specific injection-withdrawal strategy rather than identifying some optimum strategy; and treating the injection and withdrawal periods in a rather simple way. Second, this modeling framework assumes that natural gas transmission asset investment decisions are made by a single entity that seeks to minimize costs, and is able to socialize those costs over a large base of natural gas consumption. Without this assumption the breakeven price faced by a merchant-type storage provider in a competitive market setting would likely be several times larger than our assumed levelized cost. We do not model gas storage and gas transmission as separate players who each may be profit-maximizing entities interacting in a strategic setting. Depending on the environment in which gas storage may play a greater role in ensuring fuel delivery to gas-fired power plants during peak demand periods, this strategic interaction is likely to be an important determinant of cost outcomes in both gas and electricity markets.

\section{Acknowledgments}

Financial support for this work came from the US National Science Foundation under CMMI-1638331, and from the Environmental Defense Fund.

\section{References}

[1] NERC, "Special Reliability Assessment: Accommodating an Increasing Dependence on Natural Gas for Electric Power," tech. rep., PLM Interconnection, May 2013.

[2] EPIC, "Gas-electric interface study," tech. rep., Eastern Interconnect Planning Collaborative, 2014.

[3] R. D. Tabors and S. Adamson, "Measurement of energy market inefficiencies in the coordination of natural gas \& power," in System Sciences (HICSS), 2014 47th Hawaii International Conference on, pp. 2335-2343, IEEE, 2014.

[4] PLM Interconnection, "Analysis of Operational 
Events and Market Impacts During the January 2014 Cold Weather Events," tech. rep., PLM Interconnection, 2014.

[5] PJM, "PJM's evolving resource mix and system reliability," tech. rep., PJM Interconnection, March 2017.

[6] J. Zheng, Q. Wu, and Z. Jing, "Coordinated scheduling strategy to optimize conflicting benefits for daily operation of integrated electricity and gas networks," Applied Energy, vol. 192, pp. 370-381, 2017.

[7] ISO New England, "Operational Fuel Security Analysis," tech. rep., ISO New England, 2018.

[8] ICF, "Access Northeast Project," tech. rep., ICF, 2015.

[9] C. Borraz-Sánchez, R. Bent, S. Backhaus, H. Hijazi, and P. van Hentenryck, "Convex relaxations for gas expansion planning," INFORMS Journal on Computing, vol. 28, no. 4, pp. 645-656, 2016.

[10] C. Borraz-Sánchez, R. Bent, S. Backhaus, S. Blumsack, H. Hijazi, and P. van Hentenryck, "Convex optimization for joint expansion planning of natural gas and power systems," in In: Proceedings of the 49th Hawaii International Conference on System Sciences (HICSS49), pp. Kauai, HI, January 4-8, 2016.

[11] C. Borraz-Sánchez, R. Bent, P. van Hentenryck, S. Blumsack, and H. Hijazi, "Elasticity model for joint gas-grid expansion planning optimization," in In: Proceedings of the 47th Annual Conference, Symposium on Power Systems. Pipeline Simulation Interest Group (PSIG-1610), pp. Fairmont Waterfront, Vancouver, BC, Canada, May 10-13, 2016.

[12] R. Bent, S. Blumsack, P. van Hentenryck, C. Borraz Sanchez, and S. Backhaus, "Joint expansion planning for natural gas and electric transmission with endogenous market feedbacks," in Proceedings of the 51st Hawaii International Conference on System Sciences, 2018.

[13] P. Bjerksund, G. Stensland, and F. Vagstad, "Gas storage valuation: Price modelling v. optimization methods," The Energy Journal, pp. 203-227, 2011.

[14] W. Avery, G. G. Brown, J. A. Rosenkranz, and R. K. Wood, "Optimization of purchase, storage and transmission contracts for natural gas utilities," Operations Research, vol. 40, no. 3, pp. 446-462, 1992.

[15] Z. Chen and P. A. Forsyth, "A semi-lagrangian approach for natural gas storage valuation and optimal operation," SIAM Journal on Scientific Computing, vol. 30, no. 1, pp. 339-368, 2007.

[16] J. A. Bergerson and L. B. Lave, "Should we transport coal, gas, or electricity: Cost, efficiency, and environmental implications," 2005.

[17] R. D. Tabors, "Interconnection in the gcc grid: The economics of change," in System Sciences, 2009. HICSS'09. 42nd Hawaii International Conference on, pp. 1-6, IEEE, 2009.

[18] R. D. Tabors and R. Hornby, "The use of multiattribute trade-off analysis in strategic planning for an electric distribution utility: An analysis of abu dhabi distribution company," in System Sciences, 2005. HICSS'05. Proceedings of the 38th Annual Hawaii International Conference on, pp. 61b-61b, IEEE, 2005.

[19] R. D. Tabors, S. Nagendraprasad, A. Hussain, M. Ayntrazi, and J. A. Brant, "The manufacture of potable water: Case analyses of electric system alternatives," in System Science (HICSS), 2012 45th Hawaii International Conference on, pp. 19381947, IEEE, 2012.

[20] INGAA, "The Use of Liquefied Natural Gasv For Peaking Service," tech. rep., INGAA, 1996.

[21] A. Hammerschmidt, California Natural Gas Storage Utilization and Economic Analysis: Final Project Report. California Energy Commission, 2008.

[22] A. Zlotnik, L. Roald, S. Backhaus, M. Chertkov, and G. Andersson, "Coordinated scheduling for interdependent electric power and natural gas infrastructures," IEEE Transactions on Power Systems, vol. 32, no. 1, pp. 600-610, 2017. 